\title{
Measured Head CT/CTA Skin Dose and Intensive Care Unit Patient Cumulative Exposure
}

\author{
(D) R.D. Nawfel and (D) G.S. Young
}

\begin{abstract}
BACKGROUND AND PURPOSE: Estimates of cumulative CT/CTA radiation dose based on volumetric CT dose index have raised concern that neurological intensive care unit patient exposures may reach thresholds for deterministic skin injury. Because the accuracy of volumetric $C T$ dose index for this purpose in unknown, we set out to directly measure head CT and CTA peak skin dose, assess the relationship of volumetric CT dose index to measured peak skin dose, and determine whether multiple CT/CTA exposures in typical patients in the neurological intensive care unit produce cumulative doses approaching or exceeding single-dose deterministic thresholds for skin injury.
\end{abstract}

MATERIALS AND METHODS: In a prospective study from 2011-2013, nanoDot optical stimulated luminescence dosimeters were used to measure head CT/CTA peak skin dose in 52 patients (28 female, 24 male; mean age, 63 years) divided equally between 2 CT scanners. Volumetric $\mathrm{CT}$ dose index and dose-length product were recorded for each examination. Peak skin dose was also measured on an acrylic skull phantom in each scanner. A 2-tailed, unpaired $t$ test was used to compare mean patient skin doses between the 2 scanners. The measured peak skin doses were then used to calculate cumulative peak skin dose in 4 typical patients in intensive care units who received multiple CT/CTA scans.

RESULTS: Head CT/CTA peak skin dose agreed between scanners in patients and phantoms: (scanner 1 CT/CTA: patients, $39.2 \pm 3.7 \mathrm{mGy}$ and $98.9 \pm 5.3 \mathrm{mGy}$, respectively, versus phantom, $40.0 \mathrm{mGy}$ and $105.4 \mathrm{mGy}$, respectively; scanner $2 \mathrm{CT} / \mathrm{CTA}$ : patients, $42.9 \pm 9.4 \mathrm{mGy}$ and $98.8 \pm 7.4 \mathrm{mGy}$, respectively, versus phantom, $37.6 \mathrm{mGy}$ and $95.2 \mathrm{mGy}$, respectively). Volumetric CT dose index overestimated peak skin dose by a factor of 1.4-1.9 depending on examination and CT scanner. Cumulative doses in 4 patients in the intensive care unit estimated from measured CT/CTA peak skin dose ranged from 1.9-4.5 Gy.

CONCLUSIONS: Directly measured radiation skin doses from head CT/CTA patient examinations are substantially lower than volumetric CT dose index. Measured peak skin dose confirms that multiple head CT/CTA examinations in representative patients in the neurological intensive care unit may produce cumulative doses exceeding the single-dose deterministic threshold for skin injury.

ABBREVIATIONS: $C A K=$ cumulative air kerma; $\mathrm{CTDI}_{\mathrm{vol}}=$ volumetric $\mathrm{CT}$ dose index; ICU = intensive care unit; $\mathrm{PSD}=$ peak skin dose

$\mathbf{P}$

atients in the neurological intensive care unit (ICU) require rapid, accurate head CT and CTA diagnosis and monitoring to guide therapy of critical neurologic emergencies such as infarction and hemorrhage associated with high short-term risk of death or severe disability. ${ }^{1,2}$ Although 1 head CT/CTA exposure falls in the low-dose radiation range that poses no risk of deterministic effects, ${ }^{3,4}$ cumulative radiation doses from multiple head

Received June 7, 2016; accepted after revision October 13.

From the Department of Radiology (R.D.N., G.S.Y.), Brigham and Women's Hospital, Boston, Massachusetts; Department of Radiology (G.S.Y.), Dana Farber Cancer Institute, Boston, Massachusetts; and Department of Radiology (R.D.N., G.S.Y.), Harvard Medical School, Boston, Massachusetts.

The authors contributed equally to this work.

Please address correspondence to Geoffrey S. Young, MD, Department of Radiology, Brigham and Women's Hospital, 75 Francis St, Boston, MA 02115; e-mail:

gsyoung@partners.org

http://dx.doi.org/10.3174/ajnr.A5040
CT/CTA scans combined with angiography over $1-3$ weeks in patients in the ICU have been reported to range from $0.46-8.32$ Gy by using equipment-displayed dose data ${ }^{5}$ and $0.22-1.8$ Gy by using dosimeters. ${ }^{6}$ These doses approach or exceed the accepted 2-Gy single-dose deterministic threshold for skin injury, above which erythema (skin redness) and epilation (hair loss) may occur. The frequency and severity of radiation skin damage increases with dose. Transient erythema and epilation may be observed hours after a single exposure to 2-5 Gy and usually resolve by 24 hours without long-term sequelae. Latent effects, typically seen weeks after single-dose exposure to $>5 \mathrm{~Gy}$, may lead to permanent epilation and dermal atrophy or induration. Desquamation, ulceration, and necrosis are typically not seen with single doses $<10 \mathrm{~Gy} .{ }^{7,8}$ Although skin effects have been produced in a small number of patients by a series of much higher radiation dose exposures from conventional cerebral angiography and/or high- 
Table 1: Patient demographics by scanner

\begin{tabular}{lcccccc}
\hline & \multicolumn{2}{c}{ Scanner 1 (Siemens) } & & \multicolumn{2}{c}{ Scanner 2 (Toshiba) } \\
\cline { 2 - 3 } & CTA Head & Routine Head & & CTA Head & Routine Head \\
\hline No. of patients & 13 & 13 & & 13 & 13 \\
Female & 6 & 4 & & 11 & 7 \\
Male & 7 & 9 & & 2 & 6 \\
Mean age \pm SD & $67.6 \pm 11.8$ & $60.8 \pm 15.6$ & & $59.3 \pm 13.6$ & $63.1 \pm 21.6$ \\
\hline
\end{tabular}

Table 2: CT/CTA technique

\begin{tabular}{|c|c|c|c|c|c|c|c|}
\hline Scan/Phase & $\begin{array}{c}\text { Tube } \\
\text { Potential } \\
(\mathrm{kVp})\end{array}$ & $\begin{array}{c}\text { Tube } \\
\text { Current } \\
(\mathrm{mA})^{\mathrm{a}}\end{array}$ & $m A s_{\text {eff }}{ }^{a}$ & $\begin{array}{l}\text { Rot. } \\
\text { Time } \\
\text { (sec) }\end{array}$ & $\begin{array}{l}\text { Beam } \\
\text { Width } \\
\text { (mm) }\end{array}$ & Pitch & $\begin{array}{c}\text { Scan } \\
\text { Length } \\
(\mathrm{mm})\end{array}$ \\
\hline \multicolumn{8}{|l|}{ Scanner 1} \\
\hline \multicolumn{8}{|l|}{ Head CT } \\
\hline Head & 120 & 450 & 450 & 1 & $16 \times 1.2$ & & 195 \\
\hline \multicolumn{8}{|l|}{ Head CTA } \\
\hline Head & 120 & 450 & 450 & 1 & $16 \times 1.2$ & & 156 \\
\hline Premonitoring $^{\mathrm{b}}$ & 120 & 182 & 60 & 0.33 & $1 \times 10$ & & 10 \\
\hline Monitoring ${ }^{\mathrm{b}}$ & 120 & 182 & 60 & 0.33 & $1 \times 10$ & & 10 \\
\hline CTA head & 120 & 342 & 174 & 0.33 & $20 \times 0.6$ & 0.65 & 180 \\
\hline Delayed head & 120 & 585 & 450 & 0.5 & $20 \times 0.6$ & 0.65 & 171 \\
\hline \multicolumn{8}{|l|}{ Scanner 2} \\
\hline \multicolumn{8}{|l|}{ Head CT } \\
\hline Head & 120 & 250 & 286 & 0.75 & $32 \times 0.5$ & 0.65 & 160 \\
\hline Helical & 120 & 250 & 286 & 0.75 & $32 \times 0.5$ & 0.65 & 160 \\
\hline \multicolumn{8}{|l|}{ Head CTA } \\
\hline Helical & 120 & 250 & 286 & 0.75 & $32 \times 0.5$ & 0.65 & 160 \\
\hline Premonitoring ${ }^{\mathrm{b}}$ & 120 & 50 & 25 & 0.5 & $4 \times 0.5$ & & 2 \\
\hline Monitoring ${ }^{\mathrm{b}}$ & 120 & 50 & 25 & 0.5 & $4 \times 0.5$ & & 0 \\
\hline Helical & 120 & 250 & 196 & 0.5 & $64 \times 0.5$ & 0.64 & 160 \\
\hline Helical & 120 & 250 & 196 & 0.5 & $64 \times 0.5$ & 0.64 & 160 \\
\hline
\end{tabular}

Note:- $m \mathrm{~ms}_{\text {eff }}$ indicates effective $\mathrm{mAs}$; Rot., rotation.

${ }^{a}$ Tube current and $\mathrm{mAs}_{\text {eff }}$ were variable for Monitoring phase.

${ }^{\mathrm{b}} \mathrm{Scan}$ over region of chest.

Table 3: PSD and CTDI ${ }_{\text {vol }}$ from head CT/CTA by scanner (mGy)

\begin{tabular}{cccccc}
\hline & \multicolumn{2}{c}{ Scanner 1 } & & \multicolumn{2}{c}{ Scanner 2 } \\
\cline { 2 - 3 } \cline { 5 - 6 } & CTA $^{\mathbf{a}}$ & $\mathbf{C T}$ & & CTA $^{\mathbf{a}}$ & $\mathbf{C T}$ \\
\hline Patients $^{\mathrm{b}}$ & & & & \\
PSD & $98.9 \pm 5.3$ & $39.2 \pm 3.7$ & & $98.8 \pm 7.4$ & $42.9 \pm 9.4$ \\
CTDI $_{\text {vol }}$ & $187 \pm 15.1$ & $68.4 \pm 2.5$ & & $142 \pm 3.6$ & $64.6 \pm 1.5$ \\
Phantom & & & & \\
PSD & 105 & 40.0 & & 95.2 & 37.6 \\
CTDI & 167 & 68.8 & & 139 & 62.4 \\
\hline
\end{tabular}

${ }^{a} \mathrm{CTDI}_{\text {vol }}$ is total of all phases exposing head.

${ }^{\mathrm{b}}$ Dose for patient exams is given as mean \pm standard deviation.

dose CT perfusion, ${ }^{9-15}$ no skin effects have been reported in the much larger group of more typical patients in the ICU who are exposed to multiple low-dose fractions from CT/CTA without CT perfusion or repeated high-dose angiograms.

Volumetric CT dose index $\left(\mathrm{CTDI}_{\mathrm{vol}}\right)$, the most frequently used dose descriptor, does not accurately represent dose to the patient. In particular, $\mathrm{CTDI}_{\mathrm{vol}}$ has been shown to overestimate skin dose to the head, ${ }^{9,10,16-18}$ and thus is not a reliable metric for predicting risk of head CT/CTA radiation-induced skin injury. To estimate skin dose for patients in the ICU exposed to multiple CT/CTA and neurointerventional studies over a short period of time requires combining doses from multiple CT/CTA scans with angiographic dose estimates. We directly measured peak skin dose (PSD) from head CT/ CTA examinations and determined if multiple examination exposures in typical patients in the neurological ICU result in cumulative doses approaching or exceeding deterministic thresholds for skin injury.

\section{MATERIALS AND METHODS}

Institutional review board approval was obtained for this prospective study performed at the Brigham and Women's Hospital. Patient informed consent was waived by the institutional review board. Between March 2011 and June 2013, skin dose was measured by using dosimeters applied to 52 randomly selected patients, and $\mathrm{CTDI}_{\mathrm{vol}}$ was recorded during head CT/CTA examinations on 2 CT scanners representing the most disparate architectures available at our institution: a conventional 40-detector CT scanner and a 320-detector conebeam CT. Doses were measured on 26 patients scanned on scanner 1 (40 detector row CT scanner; Siemens Somatom Definition AS, Siemens, Erlangen, Germany), and 26 patients on scanner 2 (320 detector rows; Aquilion ONE CT scanner, Toshiba Medical Systems, Tokyo, Japan). On each scanner, 13 patients received a head CTA examination, and 13 patients received a routine head CT examination (Table 1). Scanning protocols are given in Tables 2 and 3. A single technologist collected examination data on each scanner, placed the dosimeters on patients, and performed the scan. CT examination dose metrics, $\mathrm{CTDI}_{\mathrm{vol}}$, and dose-length product were also recorded for each patient examination. The CTDI ${ }_{\mathrm{vol}}$ specified for CTA examinations was defined as the sum of CTDI ${ }_{\text {vol }}$ values from phases scanning over the head only; premonitoring and monitoring phases were not included in the calculation because these exposures occur to the chest rather than the head.

Patient and phantom head sizes were determined by measuring head thickness on CT images as the transverse anteroposterior $(A P)$ and lateral diameters $(L A T)$ by using the CT console software. The effective diameter was calculated as the square root of the product of the anteroposterior and lateral thickness: $(A P \times L A T)^{1 / 2}$.

\section{Study Group Skin Dose Measurement}

Doses were measured with optically stimulated luminescence dosimeters at 4 locations with respect to the surface of the patient's head. The same measurements were performed on the acrylic skull phantom (Fig $1 A$ ). The optical stimulated luminescence dosimeters used were $1 \mathrm{~cm} \times 1 \mathrm{~cm}$ aluminum oxide-based nano$\operatorname{Dots}^{19}$ (Landauer, Glenwood, Illinois) and were read by using a microStar reader (Landauer). The microStar reader was calibrated according to manufacturer's user manual recommendations by using 5 control nanoDots provided with the dosimetry system. The dosimeters were tested for reproducibility with 5 exposure measurements in air at $120 \mathrm{kVp}$. They demonstrated very good reproducibility (coefficient of variation, 0.01 ). 
Two nanoDots were placed on the surface of the patient's head: 1 on the forehead (Fig 1B) and 1 on the back of the head. Two nanoDots were also placed at locations on the head holder adjacent to the left and right temporal bone. At these lateral locations, the dosimeters were placed on the head holder instead of the patient's head to avoid disturbing the patient. Each individual patient's PSD was specified as the maximum of the 4 measurements.

\section{Skin Dose Measurement Correction Factor}

Skin dose was measured at both temporal locations on an acrylic skull phantom and corresponding locations on the head holder while using the same clinical protocols and scan parameters (Table 2). The standard filters used provide $6.8-\mathrm{mm} \mathrm{Al}$ equivalent filtration for scanner 1 and $4.8-\mathrm{mm} \mathrm{Al}$ equivalent filtration for scanner 2. Tube current modulation was used for CTA, and fixed $\mathrm{mAs}$ was used for routine head CT on both scanners. Detector configuration and collimation for CTA were 20 rows $\times 0.6 \mathrm{~mm}$ for scanner 1 and 64 rows $\times 0.5 \mathrm{~mm}$ for scanner 2 . The ratio of the skin dose measured at the temporal location of the phantom and the dose at the corresponding location on the head holder was used as a correction factor to estimate patient temporal skin dose from the head holder measurements acquired during patient ex-

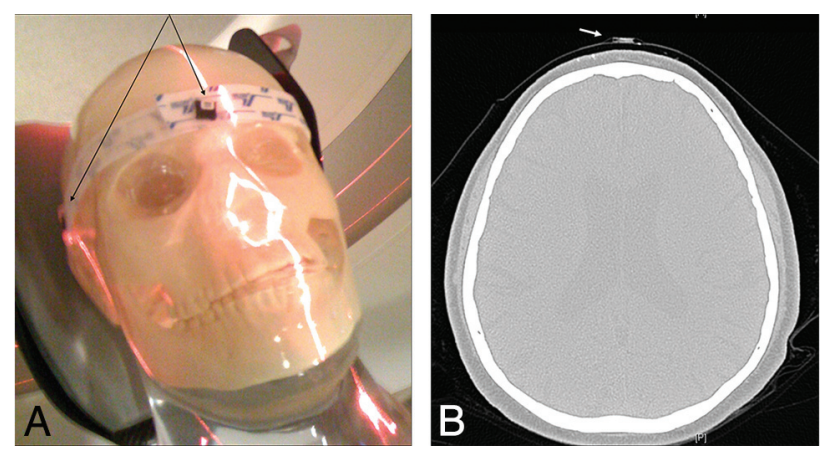

FIG 1. A, Acrylic skull phantom with nanoDots at forehead and lateral locations. B, Patient CT image with nanoDot on forehead.

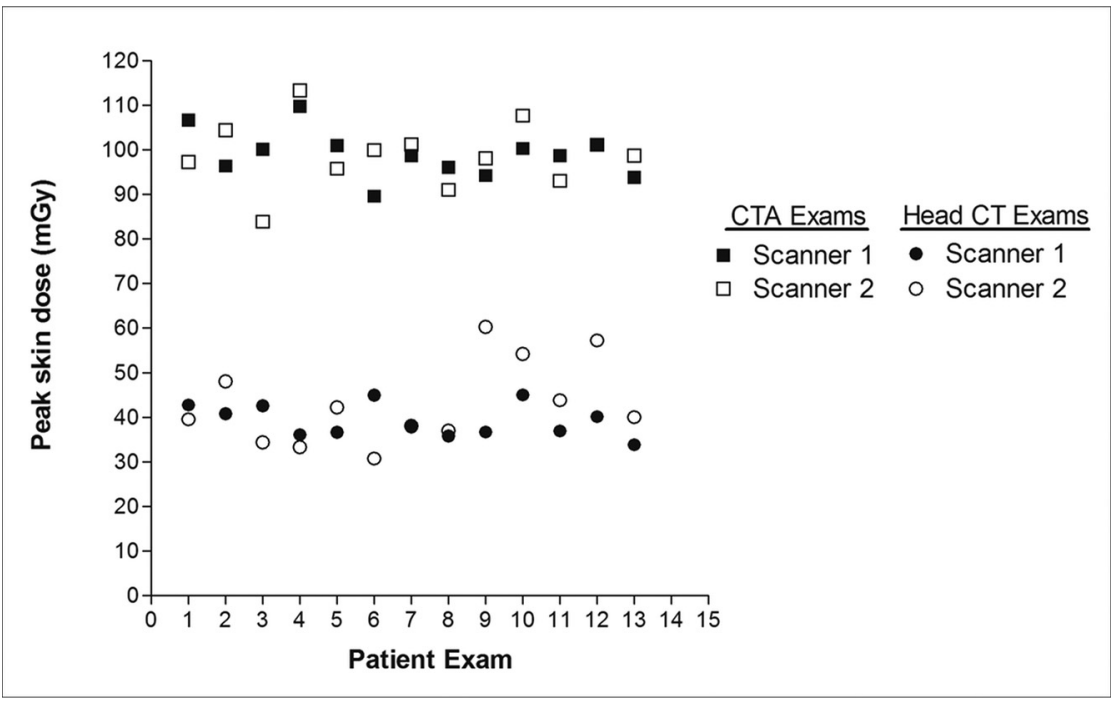

FIG 2. PSD distribution for all 52 patient examinations on both scanners. PSD was very comparable between scanners for the same patient examination. On average, the PSD for CTA examinations was more than twice that of routine head CT examinations. Note that 2 data points coincide for examination 7 (head CT) and examination 12 (head CTA). aminations. The mean correction factor was $1.33 \pm 0.10$ when averaged over all examination techniques. This factor of 1.33 was multiplied by the dose measured at the head holder locations for patient examinations.

\section{Statistical Analysis}

Mean, standard deviation, and coefficient of variation were calculated for all doses and size measurements. Correlations were also calculated to compare doses between patient groups and patient dose values with corresponding values on the phantom. A 2 -tailed, unpaired $t$ test was used to compare mean patient skin doses between the 2 scanners.

\section{Estimate of Patient Skin Dose from Neurointerventional Imaging}

Doses were measured with optical stimulated luminescence dosimeters at the same 4 locations on the surface of the acrylic skull phantom (Fig 1A) during 2 separate trials by using an interventional x-ray fluoroscopy imaging system (Innova 3100; GE Healthcare, Milwaukee, Wisconsin) with examination technique factors typically used during neurointerventional imaging, producing a total of 8 dose measurements. The maximum dose to the phantom for each assessment was specified as the PSD of the 4 measurements. The total cumulative air kerma (CAK) was also recorded from the imaging system display for each trial. This is specified as the CAK at the interventional reference point. ${ }^{20}$ The PSD to CAK ratio was calculated for each assessment. ${ }^{21}$

\section{Predicting Patient Skin Dose for ICU Clinical Examinations} Patients in the ICU who received between 12 and 27 total CT examinations and several cerebral angiograms over a period of 2-4 weeks were identified. In accordance with hospital radiation safety practice, these patients were examined for evidence of radiation-induced skin injury or hair loss. PSD measurements from the 52-patient study group were used to estimate cumulative doses to these patients in the ICU having the same examinations. For interventional angiography examinations, the PSD to CAK ratio, together with the total patient examination CAK, was used to estimate the cumulative skin dose for patients in the ICU who had previously received multiple cerebral angiograms. Total cumulative skin dose was calculated by adding the PSD estimates from CT to the skin dose estimate from angiography.

\section{RESULTS}

The maximum optical stimulated luminescence dose was measured in the forehead location in 14 patient examinations, in the left temporal location in 23 patient examinations, in the right temporal location in 15 patient examinations, and at the back of the head location in no patient examinations.

The mean PSD for CTA examinations (98.9 $\pm 5.3 \mathrm{mGy})$ and for routine head CT examinations (39.2 $\pm 3.7 \mathrm{mGy})$ per- 

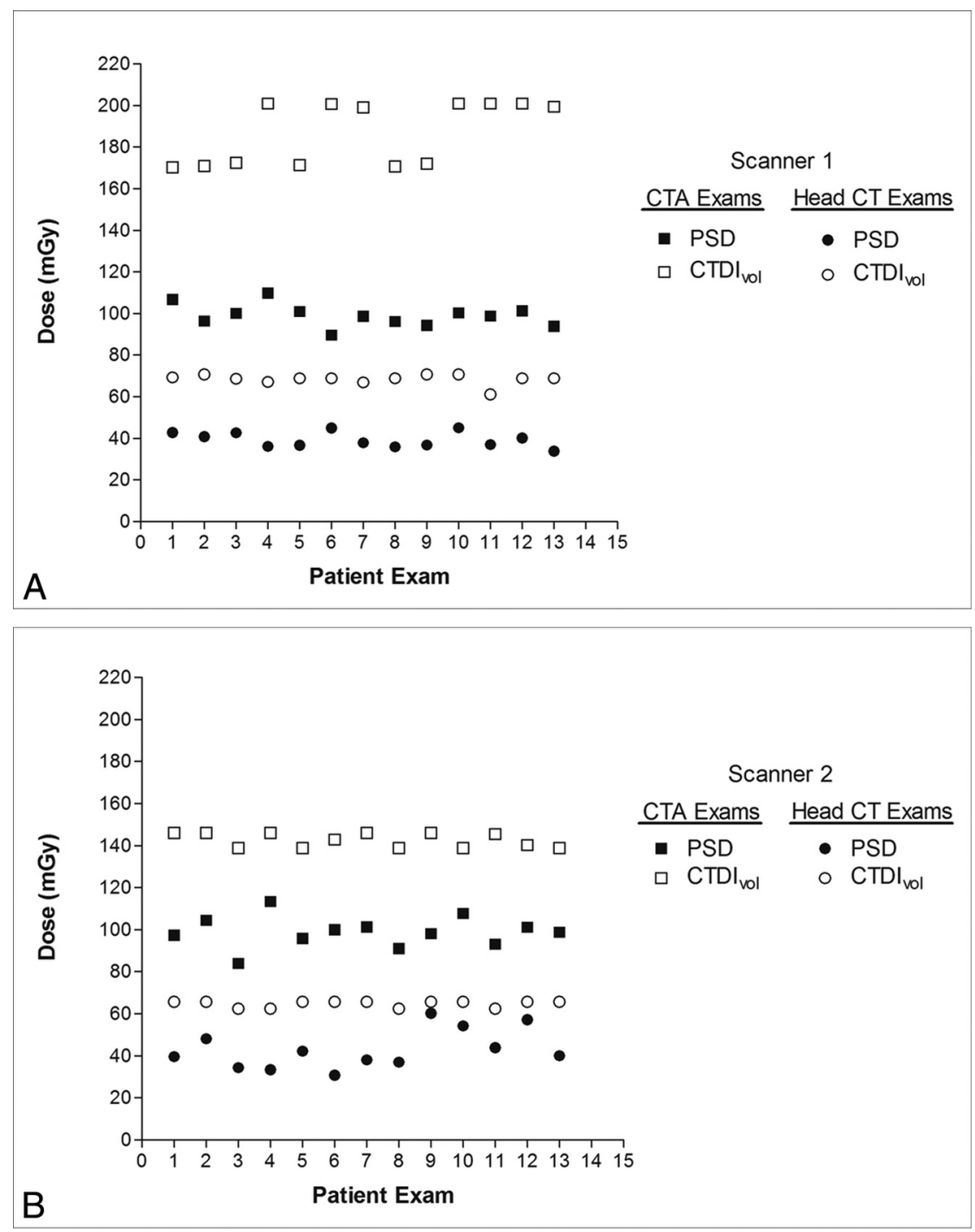

FIG 3. $A, P S D$ and $C T D I_{\text {vol }}$ for all patients examined on scanner $1 . B, P S D$ and $C T D I_{\text {vol }}$ for all patients examined on scanner 2. CTDI ${ }_{\mathrm{vol}}$ overestimated PSD for all patient examinations on each scanner for both CTA examinations and routine head CT examinations.

formed on scanner 1 agreed well with PSD measured on the acrylic head phantom: $105.4 \mathrm{mGy}$ and $40.0 \mathrm{mGy}$, respectively (Table 3). In addition, for scanner 2, the mean PSDs for CTA examinations $(98.8 \pm 7.4 \mathrm{mGy})$ and routine head CT examinations $(42.9 \pm 9.4 \mathrm{mGy}$ ) were very comparable with corresponding phantom measurements: $95.2 \mathrm{mGy}$ and $37.6 \mathrm{mGy}$, respectively. In general, the PSD for CTA examinations was slightly more than double that of routine head CT examinations (Fig 2). The difference in mean PSD for corresponding examinations on both scanners 1 and 2 were not statistically significant (CTA examinations, $P=.97$; routine head CT examinations, $P=.19$ ).

CTDI $_{\text {vol }}$ overestimated PSD for all patient examinations on both scanners 1 and 2 as illustrated in Fig 3. The mean CTDI ${ }_{\text {vol }}$ was an overestimate of the PSD for all patient examinations and phantom scans (Table 3). CTDI ${ }_{\mathrm{vol}}$ was very comparable for routine head examinations performed on both scanners; however, for CTA examinations, the CTDI ${ }_{\mathrm{vol}}$ was approximately $24 \%$ (142/ 187) lower on scanner 2 compared with scanner 1 . The difference between mean CTDI $_{\mathrm{vol}}$ for scanners 1 and 2 was statistically sig- nificant for both CTA $(P<.0001)$ and routine head CT $(P<.0001)$.

\section{Effect of Patient Head Size}

The mean effective diameters were $17.4 \pm$ $0.7 \mathrm{~cm}$ and $16.8 \pm 0.8 \mathrm{~cm}$ for patients examined on scanners 1 and 2 , respectively. The effective diameter of the phantom was $16.6 \mathrm{~cm}$. Figure 4 demonstrates the variation in PSD with patient size for all 52 patient examinations on both scanners. On neither scanner was there a significant correlation between PSD and patient head size for corresponding patient examinations (scanners 1 and 2 CTA examinations, $R^{2}=0.0008$ and $R^{2}=0.006$, respectively; scanners 1 and 2 routine head CT examinations, $R^{2}=0.0001$ and $R^{2}=0.285$, respectively).

\section{Comparison of Dose Descriptors}

Patient and phantom CTDI ${ }_{\mathrm{vol}}$ overestimated the measured PSD for both head CTA and head CT on average by $65 \%$ (range, $44 \%-89 \%$ ). The CTDI vol to PSD ratio ranged from $1.4 \pm 0.5$ to $1.9 \pm 2.8$ for the various examinations and scanners. This overestimation was statistically significant $(P<.0001)$ (Table 3$)$.

\section{Skin Dose from Neurointerventional Imaging}

The PSDs measured on the surface of the acrylic skull phantom for 2 separate assessments are given in Table 4. Using the total CAK (mGy) from each trial, the PSD to CAK ratio was determined to be 0.45 . This is consistent with literature documenting that CAK overestimates PSD. ${ }^{21}$ When the total CAK was known, the PSD to CAK ratio was used to estimate the cumulative skin dose from patient examinations performed on the same interventional imaging system by multiplying the total CAK by 0.45 .

\section{Total Patient Skin Dose from Multiple Imaging \\ Examinations}

Table 5 provides 4 examples of patients having multiple CTA and routine CT examinations of the head over a period of days to weeks. These patients also had several cerebral angiograms over the same time period. The PSD for these patients' CT examinations was calculated by multiplying the mean PSD obtained from optical stimulated luminescence measurements by the corresponding number of CTA or routine head CT examinations that the patient received. These calculated PSDs for CT were added to cumulative skin dose estimated from cerebral angiography by using the phantom measurement-based conversion above. Total cumulative skin dose ranged from 1.9-4.8 Gy for these patients. 


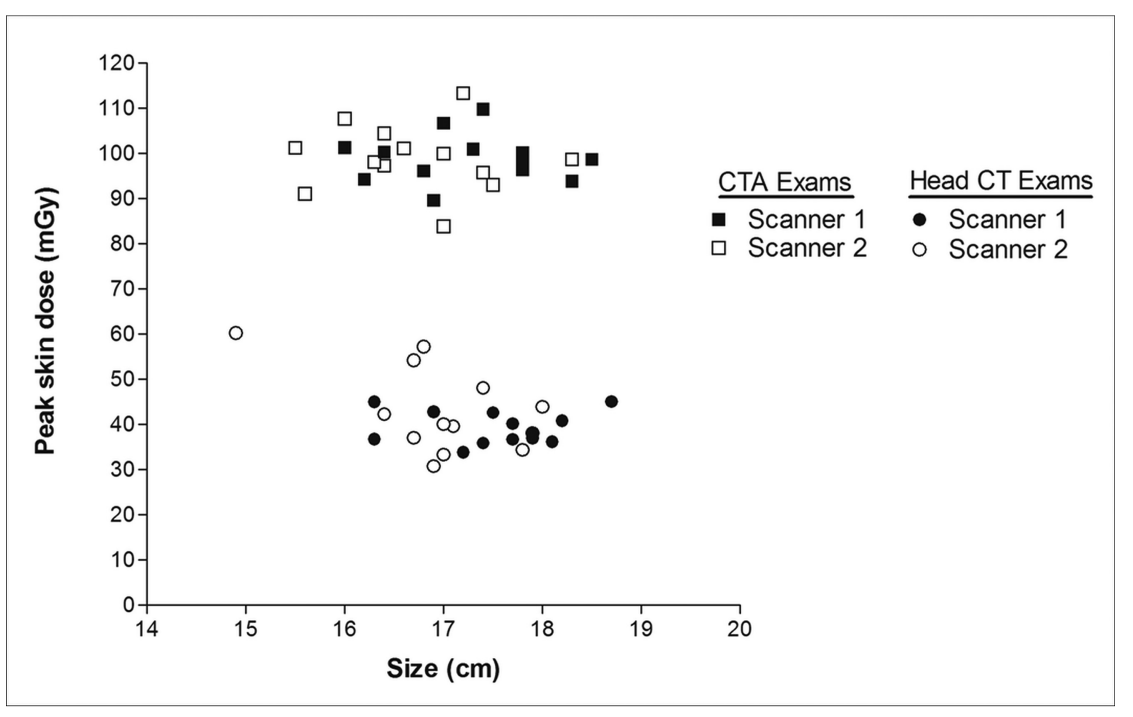

FIG 4. PSD versus patient size for all patient examinations on both scanners. Patient size is specified as the effective diameter, calculated from anteroposterior and lateral thickness measurements of patient's head. There was no significant correlation between PSD and patient head size for corresponding patient examinations on each scanner.

Table 4: Angiographic phantom CAK and PSD (mGy)

\begin{tabular}{lccc}
\hline & CAK & PSD & PSD/CAK \\
\hline Trial 1 & 73 & 33.1 & 0.45 \\
Trial 2 & 101 & 45.1 & 0.45 \\
\hline
\end{tabular}

Table 5: Illustrative ICU patient CT/CTA and cerebral angiography exposures ${ }^{\mathrm{a}}$

\begin{tabular}{lrrrr}
\hline & Patient & Patient & Patient & Patient \\
& 1 & \multicolumn{1}{c}{$\mathbf{2}$} & \multicolumn{1}{c}{$\mathbf{3}$} & \multicolumn{1}{c}{$\mathbf{4}$} \\
\hline No. of CTA & 12 & 8 & 8 & 8 \\
PSD (mGy) & 1187 & 791 & 791 & 791 \\
No. of head CT & 2 & 7 & 4 & 18 \\
PSD (mGy) & 78 & 274 & 157 & 706 \\
No. of cerebral angiograms & 11 & 9 & 2 & 14 \\
Cumulative skin dose (mGy) & 2667 & 1574 & 908 & 3261 \\
Total skin dose, all exams (mGy) & 3932 & 2639 & 1856 & 4758 \\
Time interval for all exams (days) & 30 & 18 & 16 & 31 \\
\hline a All patient CT exams performed on scanner 1. & & & \\
${ }^{b}$ Cumulative skin dose for cerebral angiography predicted from phantom measure- \\
ments.
\end{tabular}

\section{Skin Effects}

No evidence of radiation-induced skin injury or hair loss was detected in any patient.

\section{DISCUSSION}

CT and CTA are effective essential diagnostic tools for managing patients with stroke, intracranial hemorrhage, brain trauma, and other critical neurologic conditions. Typical patients undergoing multiple CTA and routine head CT scans, and in some cases cerebral angiography, may receive cumulative doses approaching accepted thresholds for deterministic radiation skin injuries such as erythema and epilation. In addition, these cumulative exposures may reach thresholds associated with cataract induction, a latent deterministic effect that may occur at single doses lower than 2 Gy. ${ }^{22}$ Importantly, the dose thresholds for these deterministic effects have primarily been studied in the setting of single exposure rather than multiple exposures separated by days or weeks. Because tissue-protective and DNA-repair effects usually occur within 24 hours, the actual threshold for induction of deterministic injury by cumulative low-dose exposures remains unknown and may be substantially higher. ${ }^{8}$ There is compelling preclinical evidence that biologic effects of low-dose and low-dose rate exposures are qualitatively distinct from well-studied highdose and high-dose rate exposures. ${ }^{4,23}$ In addition, clinical experience, as in the 4 patients from the ICU whom we studied, suggests that CT radiation-induced skin injury is very rarely observed in typical patients in the neurological ICU. Clearly, more study of the effects of cumulative CT radiation is needed to assess risk in this group. The first step in determining the actual effects of cumulative low-dose radiation is to accurately measure skin dose resulting from multiple head CT/CTA. To address this, we measured dose in a sample of patients undergoing CT/CTA on the 2 different CT scanners at our institution with the most dissimilar architectures.

In the 4 patients reported, as is typical of the adult neurological ICU population, the radiation risk is outweighed by the immediate risk of death or severe permanent disability. These patients received a large number of imaging examinations (14-30 over a period of 2-4 weeks); however, reducing the number of these examinations would compromise treatment and is likely not in the best interest of the patient. The mean PSD from the CT study groups allowed us to predict PSD from these 4 patient examinations because direct patient measurement was not available. The range in total cumulative skin dose was estimated to be 1.9-4.8 Gy, which is at or above the threshold for deterministic effects, consistent with the initial dose-measurement feasibility study by Mamourian et $\mathrm{al}^{6}$ in patients with SAH. We agree with the investigators' conclusions that radiation awareness and accurate dose assessment of these hospitalized patients are important, but caution that because these risks are much less of a concern than the immediate risk of death and severe disability in these patients, cumulative dose estimates should not be the basis for decreasing clinically indicated imaging studies or decreasing CT dose at the expense of diagnostic image quality. ${ }^{24}$

Nevertheless, surveillance for immediate or delayed skin injury may be reasonable in selected patients in the ICU who survive to leave the hospital after undergoing multiple CT examinations with high cumulative doses. Occasionally, transient erythema may be observed after coronary or cerebral angio-intervention, and epilation has been reported in a number of patients exposed to inadvertent high exposures during CT perfusion. ${ }^{7,8,10-13}$ In contrast, deterministic skin effects have not been reported in the large number of patients in the ICU exposed over the course of weeks to similar cumulative doses from multiple daily CT and CTA; however, it is possible that these effects occur but have gone undetected. Despite this remote possibility, it seems unlikely that 
such skin effects are occurring at a frequency or severity comparable with well-documented single-dose exposures. This clinical experience is consistent with the radiation biology literature showing that exposure to multiple low-dose fractions of radiation stimulates immediate DNA repair, cellular protective changes, apoptosis, and tissue effects that are substantially different from the effects of a single or several high-dose radiation exposures. ${ }^{23}$ Although these contemporary biologic data and recent epidemiologic analyses have cast substantial doubt on the validity of the linear no-threshold model for estimating low-dose radiation effects, some controversy persists in the radiation biology literature, causing concern among patients and physicians. ${ }^{3,4,23}$ Although the predicted low-level decades-delayed stochastic risks of radiation-induced carcinogenesis are very difficult to investigate clinically, direct DNA and cellular effect of radiation and rapid deterministic skin effects should be far easier to study and may help to directly address this controversy. Valid accurate estimates of patient exposure are needed as a first step toward such research.

In this study, measurement results from CT examinations demonstrated that phantom skin doses were a reasonable approximation of patient skin doses. Thus, results from phantom measurements were used when direct patient measurements were not otherwise available. Our results indicate that PSD was consistent for all patients on a particular scanner when comparing the same patient examination. This result would allow us to predict the PSD reasonably well for other patients who may receive head CTA or CT examinations on either of these scanners. In contrast, the mean $\mathrm{CTDI}_{\mathrm{vol}}$ overestimated the mean PSD by a factor ranging from $1.4 \pm 0.5$ to $1.9 \pm 2.8$ depending on the specific CT protocol and scanner, and there is some interpatient variation in the relationship of CTDI $_{\mathrm{vol}}$ and PSD (Fig 3B). These findings confirm that $\mathrm{CTDI}_{\mathrm{vol}}$ alone is inadequate as an indicator of patient radiation dose and potential for radiation-induced deterministic effects. As such, though a correction factor for $\mathrm{CTDI}_{\mathrm{vol}}$ specific to each scanner and protocol would be possible, we chose not to use $\mathrm{CTDI}_{\mathrm{vol}}$, but instead to calculate cumulative individual patient dose for our 4 patients from the ICU directly from PSD measurements on the relevant scanner and protocol.

Prediction of patient skin dose in a single patient undergoing multiple scans by using dose measurements on multiple patients is a limitation of this study. This was unavoidable because of the logistical challenges of placing and retrieving the dosimeters on patients who are critically ill and may be scanned at any time of day or night. Our data indicate that there was a high degree of consistency between PSD on different scanners for the same CT examination protocol and that the head phantom served as an acceptable alternative to patient dose measurements, particularly for assessment of skin dose during cerebral angiography. For these reasons, we believe our method provided a reasonable initial basis for dose quantification. Although direct measurements on the same patient having multiple examinations would have been a more valid means of data collection, this would have been extremely cumbersome in the most critically ill patients. Although our measurement results were useful in calculating the PSD for examinations and protocols on our scanners, the results of this study reflect the scanners and imaging protocols specifically at our institution.

\section{CONCLUSIONS}

Directly measured head CT/CTA PSD was reasonably consistent for a given examination across patients and CT scanners. Using these scanner- and protocol-specific PSD measurements to predict doses to 4 typical patients in the neurological ICU exposed to multiple CT/CTA examinations, we found cumulative skin doses ranged from 1.9-4.8 Gy, at or above the threshold for single-dose deterministic skin effects, but we observed no skin effects. Further study correlating diagnostic CT exposure in patients in the ICU with direct cellular, DNA, and deterministic skin effects is needed to investigate what if any risk these cumulative exposures impart. Meanwhile, cumulative dose estimates should not be used to justify reduction in individual CT scan dose at the expense of diagnostic image quality or to limit the number of otherwise clinically indicated scans in critically ill patients in the neurological ICU.

Disclosures: Geoffrey Young —UNRELATED: Grants: Dr. Young received no salary or direct research support relevant to the research reported in this paper. Toshiba Medical Systems provided research support to the Department of Radiology of Brigham and Women's Hospital to support research on neurologic CT/CTA unrelated to radiation dose or the research reported in this paper.

\section{REFERENCES}

1. Mokin M, Kass-Hout T, Kass-Hout O, et al. Intravenous heparin for the treatment of intraluminal thrombus in patients with acute ischemic stroke: a case series. J Neurointerv Surg 2013;5:144-50 CrossRef Medline

2. Camargo EC, Furie KL, Singhal AB, et al. Acute brain infarct: detection and delineation with $\mathrm{CT}$ angiographic source images versus nonenhanced CT scans. Radiology 2007;244:541-48 CrossRef Medline

3. Wall BF, Kendall GM, Edwards AA, et al. What are the risks from medical x-rays and other low dose radiation? Br J Radiol 2006;79: 285-94 CrossRef Medline

4. Tubiana M, Feinendegen LE, Yang C, et al. The linear no-threshold relationship is inconsistent with radiation biologic and experimental data. Radiology 2009;251:13-22 CrossRef Medline

5. Wong JM, Ho AL, Lin N, et al. Radiation exposure in patients with subarachnoid hemorrhage: a quality improvement target. J Neurosurg 2013;119:215-20 CrossRef Medline

6. Mamourian AC, Young H, Stiefel MF. Cumulative radiation dose in patients admitted with subarachnoid hemorrhage: a prospective study using a self-developing film badge. AJNR Am J Neuroradiol 2010;31:1787-90 CrossRef Medline

7. Koenig TR, WolffD, Mettler FA, et al. Skin injuries from fluoroscopically guided procedures: part 1 , characteristics of radiation injury. AJR Am J Roentgenol 2001;177:3-11 CrossRef Medline

8. Balter S, Hopewell JW, Miller DL, et al. Fluoroscopically guided interventional procedures: a review of radiation effects on patients' skin and hair. Radiology 2010;254:326-41 CrossRef Medline

9. Zhang D, Cagnon CH, Villablanca JP, et al. Estimating peak skin and eye lens dose from neuroperfusion examinations: use of Monte Carlo based simulations and comparisons to CTDI ${ }_{\text {vol, }}$ AAPM Report No. 111, and ImPACT dosimetry tool values. Med Phys 2013; 40:091901 CrossRef Medline

10. Zhang D, Cagnon CH, Villablanca JP, et al. Peak skin and eye lens radiation dose from brain perfusion CT based on Monte Carlo simulation. AJR Am J Roentgenol 2012;198:412-17 CrossRef Medline

11. Hoang JK, Wang C, Frush DP, et al. Estimation of radiation exposure for brain perfusion CT: standard protocol compared with deviations in protocol. AJR Am J Roentgenol 2013;201:W730-34 CrossRef Medline

12. Imanishi Y, Fukui A, Niimi H, et al. Radiation-induced temporary hair loss as a radiation damage only occurring in patients who had 
the combination of MDCT and DSA. Eur Radiol 2005;15:41-46 CrossRef Medline

13. Hirata M, Sugawara $Y$, Fukutomi $Y$, et al. Measurement of radiation dose in cerebral CT perfusion study. Radiat Med 2005;23:97-103 Medline

14. Diekmann S, Siebert E, Juran R, et al. Dose exposure of patients undergoing comprehensive stroke imaging by multidetector-row CT: comparison of 320-detector row and 64-detector row CT scanners. AJNR Am J Neuroradiol 2010;31:1003-09 CrossRef Medline

15. Yamauchi-Kawara C, Fujii K, Aoyama T, et al. Radiation dose evaluation in multidetector-row CT imaging for acute stroke with an anthropomorphic phantom. Br J Radiol 2010;83:1029-41 CrossRef Medline

16. Bauhs JA, Vrieze TJ, Primak AN, et al. CT dosimetry: comparison of measurement techniques and devices. Radiographics 2008;28: 245-53 CrossRef Medline

17. McCollough $\mathrm{CH}$, Leng S, Yu L, et al. CT dose index and patient dose: they are not the same thing. Radiology 2011;259:311-16 CrossRef Medline
18. Rong X, Cody D. How accurate is estimating CT skin dose based on CTDI? Med Phys 2010;37:3463 CrossRef

19. Patient Monitoring Solutions nanoDot Dosimeter [technical specifications]. Glenwood, IL: Landauer Inc; 2010

20. Stecker MS, Balter S, Towbin RB, et al. Guidelines for patient radiation dose management. J Vasc Interv Radiol 2009;20:S263-73 CrossRef Medline

21. Miller DL, Balter S, Cole PE, et al. Radiation doses in interventional radiology procedures: the RAD-IR study: part II: skin dose. $J$ Vasc Interv Radiol 2003;14:977-90 CrossRef Medline

22. Miller DL, Schueler BA, Balter S. New recommendations for occupational radiation protection. J Am Coll Radiol 2012;9:366-68 CrossRef

23. Averbeck D. Does scientific evidence support a change from the LNT model for low-dose radiation risk extrapolation? Health Phys 2009;97:493-504 CrossRef Medline

24. Durand DJ. A rational approach to the clinical use of cumulative effective dose estimates. AJR Am J Roentgenol 2011;197:160-62 CrossRef Medline 